\title{
Intention-behaviour consistency: effects of consideration set size, involvement and need for cognition
}

\author{
RIK G. M. PIETERS \\ Tilburg University, The Netherlands \\ and \\ BAS VERPLANKEN \\ University of Nijmegen, The Netherlands
}

\begin{abstract}
This study focuses on the strength of the relationship between behavioural intentions and actual behaviour in a multi-alternative choice context. Two separate moderating processes of intention-behaviour consistency were hypothesized, i.e. the amount of reasoning during intention formation, and the degree of confidence in the intention. Involvement (as an issue-specific factor), and need for cognition (as an individual difference factor) were investigated as antecedents of amount of reasoning. Confidence in the intention was predicted from the size of the consideration set (i.e. the number of alternatives that one considers for choice), and involvement. The study comprised a longitudinal two-wave survey conducted before and after national elections in The Netherlands, in which pre-election voting intentions were compared with actual voting behaviour. A high degree of intention-behaviour consistency was found, which was significantly related to both amount of reasoning and confidence. The expected relations were found. The results extend current process models of attitude-behaviour relations. Furthermore, the results indicate that processes related to the consideration set size and content account for variance in intention-behaviour consistency in choice contexts that cannot be accounted for by traditional attitude-behaviour perspectives.
\end{abstract}

\section{INTRODUCTION}

In the last two decades much progress has been made in understanding the conditions and underlining processes that moderate the relationship between attitudes and

Addressee for correspondence: Rik Pieters, Tilburg University, Department of Business Administration, PO Box 90153, 5000 LE Tilburg, The Netherlands.

This study was financially supported by Research International Rotterdam. We thank Réne van der Giessen for his help with the data collection, and two anonymous reviewers of the European Journal of Social Psychology for their insightful comments on a previous version. 
behaviour (Fazio, 1990). However, most of this research has focused either on the relationship between attitudes and behavioural intentions, or on the direct link between attitude and behaviour. The intention-behaviour relationship has received less attention. Furthermore, the behaviour under scrutiny mostly concerns the frequency of performing one particular act (e.g. attending classes), or the choice between two mutually exclusive alternatives (e.g. breast-feeding versus bottle-feeding of infants). Yet, in real life, people often choose from larger sets of alternatives, for example when selecting job candidates, choosing holiday destinations, voting for one from a set of parties, or when choosing brands. In this study factors that are related to intentionbehaviour consistency in a multi-alternative choice context are focused upon.

Although attitudes may sometimes be directly linked to behaviour without a mediating role of behavioural intentions (Bentler and Speckart, 1979), intentions have generally been found to be important mediators of the attitude-behaviour relationship, as is postulated in Ajzen and Fishbein's (1980) model of reasoned action. Compared to the attitude-behaviour relation, there has been relatively little theorizing about the intention-behaviour relation. In the theory of reasoned action, for instance, behavioural intentions are assumed to be approximately equivalent to subsequent behaviour, suggesting that there are no processes of theoretical interest that mediate the intention-behaviour relationship. Explanations of intention-behaviour inconsistency have often referred to methodological issues, for instance the level of specificity of measurements of intention and behaviour (Ajzen and Fishbein, 1977), the length of the time interval between the measurements of intentions and behaviour (Davidson and Jaccard, 1979), or whether intentions should be formulated as plans or as expectations (Sheppard, Hartwick and Warshaw, 1988; Warshaw and Davis, 1985).

Recently, the processes that may intervene between the formation of an intention and the performance of behaviour have gained attention. Bagozzi and Warshaw (1990) propose a process of trying between the formation of intention to attain some goal and actual goal attainment. The more people try to reach a goal, the higher the likelihood that they perform the relevant behaviours, and attain the goal. Hence, inconsistency may be due to a failure to perform the behaviours that correspond with the intentions. In a related approach, Gollwitzer (1993) distinguishes four phases in the intentionbehaviour course, i.e. a predecisional phase, in which wishes and desires are deliberated, a post-decisional but pre-actional phase, in which planning occurs to make wishes come true, an actional phase, in which actions for goal attainment are initiated, and an evaluation phase, in which outcomes are attained and evaluated. Gollwitzer (1993) distinguishes goal intentions, which refer to a desired end-state, from implementation intentions, which specify in relatively great detail when and how the attainment of the desired goal will be reached. Goal intentions that are not accompanied by implementation intentions are less likely to lead to the corresponding behaviour than goal intentions that are accompanied by a proper set of implementation intentions.

However, when people change their mind between the formation of an intention and the performance of the behaviour, the original intention also does not correspond any more with the actual behaviour that is performed. Belk (1985) has suggested several sources of changes that may occur in the time interval between intention formation and behaviour expression, i.e. sources within the person, perception of choice objects, affect aroused by choice objects, normative beliefs, and the situation in which an act is performed. In the present study we investigate specific conditions that cause people to keep or change their intentions between intention formation and actual expression of 
behaviour. First, building on the literature concerning the role of involvement in the attitude-behaviour relationship, the motivation to think and deliberate about one's intention is examined as a possible moderator of intention-behaviour consistency. Second, we focus on the number of behavioural options that people consider for choice as a possible source of intention-behaviour consistency. The processes through which these factors impact on intention-behaviour consistency are analysed in-depth.

\section{Amount of reasoning: involvement and need for cognition}

Our first focus in the present study is on a possible moderator of the intentionbehaviour relationship that is well-researched with respect to the attitude-intention and attitude-behaviour relationships, i.e. the amount of cognitive elaboration one engages in during the formation of an attitude and, in our case, a behavioural intention. A large body of research indicates that attitudes that are formed or changed on the basis of relatively extensive deliberation and cognitive elaboration of information are more strongly related to behavioural intentions and behaviour than attitudes that are based on cognitively less effortful processing. Dual-process models such as the elaboration likelihood model of persuasion (ELM; Petty and Cacioppo, 1986), and the heuristic-systematic model (HSM; Chaiken, 1987), describe these processes in detail. These models specify that, when an individual is sufficiently motivated and able to process information, attitudes tend to be formed or changed through relatively effortful processing or reasoning, for example by scrutinizing issue-relevant argumentations ( $c f$. the central route in the ELM, and systematic processing in the HSM). On the other hand, when motivation is low and/or the ability to engage in extensive processing is absent, attitudes tend to be adopted on the basis of simple cues in the persuasion context (the peripheral route in the ELM), or simple decision rules (heuristic processing in the HSM). Important for the present discussion is that attitudes that are formed via central route, systematic, or reasoned processing are more predictive of subsequent behaviour than attitudes that are based on simple cues or heuristic processing.

In an illustrative study, Petty, Cacioppo, and Schumann (1983) found that behavioural intentions of consumers who were highly involved with respect to disposable razor blades were more consistent with their attitudes than behavioural intentions of low-involvement consumers. In that study personal relevance of the object of attitude motivated subjects to engage in reasoning about the issue. Generally, the more involved individuals are with an issue, the more likely it is that they will allocate cognitive effort to understanding the issue and to making a decision. Consequently, high levels of involvement are related to a high degree of consistency between attitudes and intentions (Krosnick, 1988; Petty et al., 1983; Verplanken, 1989). Intentions that are thus formed under conditions of high cognitive elaboration can be expected to be more consistent with subsequent behaviour ( $c f$. Bagozzi and $\mathrm{Yi}, 1989$ ).

In addition to issue-related motivation to engage in processes of reasoning (i.e. involvement), there is also evidence to suggest that individual differences in a chronic tendency to engage in reasoning exist (i.e. need for cognition; Cacioppo and Petty, 1982), which are related to attitude-behaviour consistency. Cacioppo, Petty, Kao and Rodriguez (1986) found that attitudes toward presidential candidates of persons high in need for cognition are more predictive of subsequent voting behaviour than lowneed-for-cognition voters' attitudes. 
In sum, there is substantial evidence to suggest that amount of reasoning moderates the relationship between attitudes, intentions, and behaviour. This raises the question at which part of the attitude-behaviour route amount of reasoning is actually effective as a moderating factor. On the one hand the moderating quality of amount of reasoning may operate exclusively on the attitude-intention relationship, and not on the intention-behaviour relationship. If this is the case, amount of reasoning moderates the attitude-behaviour relationship because of its impact on the attitude-intention relationship. Then, amount of reasoning would not affect the intention-behaviour link. On the other hand, when similar moderating processes related to amount of reasoning operate in the intention-behaviour relationship as they do in the attitude-intention relationship, a moderating effect of amount of reasoning in the intention-behaviour relation will be found. This latter possibility is hypothesized in the present study.

\section{Consideration set size}

Research indicates that in multi-alternative choice contexts decision makers tend to use a two-stage procedure in order to arrive at a choice (e.g. Bettman and Park, 1980; Gertzen, 1992). In the first stage, the total set of alternatives a person is aware of (i.e. awareness set) is reduced to a smaller set of alternatives, which are seriously considered for choice. This latter set is referred to as consideration set (Howard and Sheth, 1969). For example, suppose a liberal voter in an election can choose from six political parties (i.e. the awareness set). He or she may immediately skip three parties because their political positions are too discrepant from a liberal point of view. The three parties left may have programmes that are acceptable to liberals, and may thus be considered for voting (i.e. the consideration set). Selection of promising alternatives in the first stage is frequently accomplished by using noncompensatory selection rules such as conjunctive, lexicographic, or elimination-by-aspects rules. In the second stage compensatory rules, as represented in subjective-expected utility and multi-attribute models, are dominant in selecting the most preferred option from the consideration set (Bettman, 1979).

Undoubtedly, dynamics that guide attitude-behaviour relationships in a multialternative context are different from those involving single-option behaviours. It is likely that the size of the consideration set is an important factor in the decisionmaking process. In particular, we expect that the size of the consideration set is related to the degree of confidence a decision maker has in the behavioural intention to choose a particular alternative from the set. The larger the consideration set, the higher the competition between alternatives will be. When one forms an intention to choose one particular option from a large consideration set, other choice alternatives also meet the minimum requirements that the person has set. In that case, the likelihood is high that between formation of the original intention and expression of the behaviour, the person changes his or her mind, and forms an intention to choose a different alternative. Hence, it is likely that the larger the size of the consideration set, the less confidence the person will have in the intention to choose one particular alternative from the set, and the higher the likelihood of observing a discrepancy between the original intention and the actual behaviour.

This leads to the hypothesis that consideration set size, through its impact on confidence, is negatively related to intention-behaviour consistency. Evidence that 
confidence, in general, is related to consistency comes, for example, from early studies on direct experience. Fazio and Zanna (1978) found that attitudes that are formed by direct experience with the object of behaviour are held with more confidence compared to attitudes that were not based on direct experience. Important for the present discussion is that they demonstrated that confidence is causally related to attitudebehaviour consistency. Those who are relatively confident in their attitude expressed higher degrees of consistency than those with less confidence. Like direct experience, other antecedents of confidence may thus moderate attitude-behaviour consistency. In the present study consideration set size is hypothesized as such an antecedent, when choice is made in a multi-alternative context.

In addition to consideration set size, involvement may also be related to confidence. It is likely that people who are highly involved with an issue will hold their intentions with respect to the issue with more confidence than other people do. Sherif and Hovland (1961) found that people who are highly involved, compared to people who are less involved, tend to reject significantly more statements about an issue (i.e. have a larger latitude of rejection), and tend to accept significantly less statements that deviate from their own specific position on the issue (i.e. have a smaller latitude of acceptance). Fazio and Zanna (1978) found that latitude of rejection was positively correlated with confidence in one's attitude, which in turn moderated attitude-behaviour consistency. This indicates that people who are involved will be more confident in their intention.

\section{Intention-behaviour consistency in a multi-alternative context}

The previous analysis leads to a conceptual model of key moderating factors in the intention-behaviour relationship concerning multi-alternative choices. The conceptual model is graphically represented in Figure 1, and specific hypotheses that derive from the model are summarized below. First, we expect that the amount of reasoning during the formation of intentions moderates intention-behaviour consistency. Involvement (as an issue-specific factor), and need for cognition(as an individual difference factor) are hypothesized as antecedents of amount of reasoning. Second, we expect that confidence in one's behavioural intention is a separate moderator of intention-behaviour consistency. We hypothesize consideration set size and involvement as antecedents of confidence. The conceptual model thus distinguishes two direct moderators of the intention-behaviour relationship, and three antecedents of these moderators, with involvement having an impact on both moderators.

National elections for parliament in The Netherlands that were held in 1989 were used as a context of a two-wave study, in which literal consistency between voting intentions and voting behaviour were recorded. These elections comprised a choice between a sizable number of parties, which provided an opportunity to investigate intention-behaviour consistency in a multi-alternative context.

\section{METHOD}

\section{Design, sampling, and procedure}

The study was conducted in two waves, using a mixed-mode data collection. The first wave comprised a mail survey. In the second wave telephone interviews were conducted. The mixed-mode design was used to minimize the likelihood that people were 
aware of the fact that they participated in a single study, and to allow a swift collection of data immediately after the election day.

The mail questionnaire (wave 1) was sent to 500 households in The Netherlands eight weeks before the election day. Addresses were randomly drawn from the telephone directory. The cover letter, which contained a university letter head, asked to have the questionnaire completed by 'the person in the household who is allowed to vote and who was the last to celebrate his or her birthday'. A reminder postcard was sent to all sampled addresses after 8 days. A total of 237 questionnaires were returned within five weeks (i.e. response rate $=47.4$ per cent) .

During the first two days after the election day a random sample of 190 people, from the 500 people who had been sampled in the first wave, were sampled for the second wave, and were contacted for a telephone interview by professional female interviewers of a market research company. One hundred and sixty three people accepted to be interviewed (i.e. 85.6 per cent). The final response rate for the group that was sampled twice therefore amounts to 40.7 per cent. In addition, a small sample of 25 people, who were not sampled in the first wave, were randomly drawn from the telephone directory and contacted, of which 21 agreed to be interviewed. The sample in wave 2 thus comprised three subgroups: persons who had responded in wave $1(n=139)$, persons who had been sampled for wave 1 , but who had not responded in wave $1(n=24)$, and persons who had not been sampled at all for wave $1(n=21)$. By including these different groups we could examine indications of nonresponse bias and measurement effects in addition to tests of the hypotheses.

\section{Measures in wave 1}

The questionnaire of wave 1 consisted of a 4-page booklet. In the elections five major parties participated ${ }^{1}$. Intentions to vote for one of these parties was measured by the item worded 'Please indicate which party you intend to vote for in the national elections in September'. Responses were indicated as follows: ' $I$ intend to vote for: [X]', 'I do not intend to vote', and 'I really don't know yet'. In the questionnaire '[X]' was replaced by the name of each of the five major parties, and by a sixth response alternative labelled 'other parties'. Each response alternative was accompanied by a small box, which could be checked.

Confidence in one's voting intention was assessed with the item 'How confident are you that you actually will vote for the party you just indicated?' Responses were provided on a 5-point scale, ranging from 'I am absolutely not confident' to 'I am completely confident' $(M=4.29 ; S . D .=0.93)$.

The size and content of the consideration set was measured by asking 'Which parties or party do you consider to vote for in the national elections in September? You may indicate more than one party'. The response alternatives were the five major parties, and the alternative 'other parties'. The consideration set size is the number of parties that were checked. This measure thus varies between 0 and 6 . The mean consideration set size amounts to $1.72(S . D .=0.93)$. Consideration set size is largest among those who do not know yet which party to vote for $(M=2.50)$, and smallest when one does not intend to vote at all $(M=1.33)$.

\footnotetext{
${ }^{1}$ The actual number of parties on the bill was much larger. However, most of the remaining parties were very small, or only locally present. We restricted our measures to the five major parties that actually played a role in these elections.
} 
Two indicators of amount of reasoning were included: self-reported amount of reasoning about the elections a person had been engaged in (i.e. 'How much have you been thinking about the coming elections in September?'), and self-reported extent to which the elections were a topic in conversations with other people (i.e. 'I almost never talk with other people about the coming elections in September'). The items were accompanied by 5-point Likert-type agree-disagree scales. After reversal of the second item the measure of amount of reasoning was constructed by averaging across the items $(M=3.18 ; S . D$. $=0.82 ; r=0.43, p<0.001, n=226)$.

Involvement with the elections was measured with two items: 'The coming elections are very important for me', and 'I don't care who will win the elections in September', accompanied by 5-point Likert-type agree-disagree scales. The items were averaged after proper scoring to form the involvement measure $(M=4.09 ; S . D .=0.62 ; r=0.33, p$ $<0.001, n=231$ ).

Need for cognition was measured by a 15 -item Dutch version of Cacioppo, Petty and Kao's (1984) scale. After reversal of negatively worded items a need for cognition scale was formed by averaging across items $(M=3.48 ; S . D .=0.50$; coefficient alpha $=0.79)$.

\section{Measures in wave 2}

Among other questions in the alleged marker research interview, voting behaviour was recorded by asking respondents which party they had voted for in the national elections. Responses were coded in one of eight alternatives, i.e.: the five major parties, 'other parties', 'did not vote', and 'refuses to tell'. In addition, respondents were asked to indicate when they knew which party they were going to vote (decision moment). Responses to this open question were coded by the interviewers into one of 10 alternatives, i.e. in the voting booth, on the election day, one day, a few days, about a week, about two weeks, about a month, a few months, longer than a few months before the elections, and 'don't know/other responses'.

\section{RESULTS}

The presence of nonresponse bias and measurement effects was investigated with respect to voting behaviour and self-reports of the decision moment, as measured in wave 2. Nonresponse bias is revealed if significant differences exist in wave 2 between the respondents and nonrespondents in wave 1 . There were neither significant differences in party choice, $\chi^{2}(6)=2.83$, n.s., nor in decision moment, $t(157)=0.06$, n.s.

Measurement effects occur when participation in wave 1 affects responses in wave 2. Therefore, voting behaviour and decision moment of respondents in wave 2 who were not sampled for wave 1 were tested against those who responded in both waves. There were no indications of measurement effects in voting behaviour, $\chi^{2}(6)=4.36$, n.s., and decision moment, $t(155)=1.63$, n.s.

\section{Intention-behaviour consistency}

To determine intention-behaviour consistency, only those people who had responded in both waves were considered $(n=139)$. Eleven people had either a missing value on the intention measure in wave 1 (i.e. no response, or a response in the category 'I really 
don't know yet'), or refused to indicate their voting behavior in wave 2, or both, and were thus excluded from the analyses on consistency. Each of the remaining 128 respondents were classified either as literally consistent if their voting intention expressed in wave 1 and their self-reported behaviour in wave 2 were exactly the same, or as literally inconsistent, if their voting intention and self-reported behaviour were not the same. A total of 109 out of 128 respondents ( 85 per cent) were classified as literally consistent. Thus, respondents exhibited a very high degree of intention-behaviour consistency.

To test the hypotheses that amount of reasoning and confidence moderate intention-behaviour consistency, logistic regression analyses were performed (LOGIT), in which literal consistency between voting intention (wave 1) and behaviour (wave 2) was regressed on self-reported amount of reasoning and confidence in one's intention (both expressed in wave 1). The results are presented in the top panel of Table 1 under the heading 'Model 1'. The results indicate that, as hypothesized, both amount of reasoning and confidence moderate intention--behaviour consistency statistically significantly, which is indicated by a significant chi-square of the analysis, and by the significant values of the Wald statistics for both predictors' regression weights.

In the bottom panel of Table 1 crosstabulations of the two moderating factors with literal consistency are presented. To ease the interpretation of the results, the moderating variables are dichotomized (the original scale values underlying the dichotomization are presented between parentheses). Fifteen out of the 19 inconsistent respondents ( 79 per cent) were less than completely confident about their voting intention in wave 1 , while only 38 out of the 108 consistent respondents ( 35 per cent) were less than completely confident. Also, 10 out of the 19 inconsistent respondents ( 53 per cent) reported little reasoning prior to forming their voting intention, while only 27 out of the 108 consistent respondents ( 25 per cent) reported little reasoning. In both cases, the proportion of intention-behaviour inconsistency is significantly higher for

Table 1. Logistic regression analyses on intention-behaviour consistency

\begin{tabular}{|c|c|c|c|c|}
\hline Predictors/fit & Regression weight & \multicolumn{2}{|c|}{$\begin{array}{l}\text { Wald statistics } \\
\qquad(d f=1)\end{array}$} & $p$ \\
\hline \multicolumn{5}{|l|}{ Model 1} \\
\hline Confidence & 0.789 & \multicolumn{2}{|c|}{7.81} & 0.005 \\
\hline Amount of reasoning & 0.717 & \multirow{2}{*}{\multicolumn{2}{|c|}{$\begin{array}{l}4.21 \\
5.92\end{array}$}} & 0.040 \\
\hline (Constant) & -3.509 & & & 0.015 \\
\hline$\chi^{2}(2)$ & 14.81 & & $<0.001$ \\
\hline \\
\hline$\chi^{2}(5)$ & \multicolumn{3}{|l|}{19.40} & $<0.001$ \\
\hline \multicolumn{5}{|l|}{ Model 2 - Model 1} \\
\hline$\chi^{2}(3)$ & \multicolumn{2}{|l|}{4.59} & & 0.200 \\
\hline & \multicolumn{2}{|c|}{ Confidence } & \multicolumn{2}{|c|}{ Amount of reasoning } \\
\hline & $\begin{array}{l}\text { Less than } \\
\text { completely } \\
\text { confident } \\
(1-4)\end{array}$ & $\begin{array}{l}\text { Completely } \\
\text { confident } \\
\text { (5) }\end{array}$ & $\begin{array}{c}\text { Relatively } \\
\text { little } \\
(1.0-2.5)\end{array}$ & $\begin{array}{c}\text { Relatively } \\
\text { much } \\
(3.0-5.0)\end{array}$ \\
\hline Inconsistent & 15 & 4 & 10 & 9 \\
\hline Literally consistent & 38 & 70 & 27 & 81 \\
\hline
\end{tabular}


those who were less than completely confident (z-test for difference between proportions is 3.56, $p<0.001$ ), and for those who had engaged in little reasoning ( $z$-test for difference between proportions is $2.44, p<0.05$ ).

To test whether effects of involvement, need for cognition, and consideration set size on intention-behaviour consistency are mediated by amount of reasoning and confidence respectively, an additional logistic regression was performed. In this analysis, literal consistency was regressed on all five relevant factors. The results are presented in the upper panel of Table 1 under the heading 'Model 2'. If amount of reasoning and confidence completely mediate the effects of involvement, need for cognition, and consideration set size, the addition of the latter three components to the logistic regression equation containing amount of reasoning and confidence will leave the overall fit unchanged, as is indicated by the difference in chi-squares of Model 1 and Model 2. Clearly, addition of involvement, need for cognition, and consideration set size does not significantly improve the fit of the model, $\chi^{2}$-difference $=4.59, d f=3$, n.s. ${ }^{2}$.

\section{Overall test}

To provide an overall test of the model outlined in the theory section, a path analysis was performed on the sample variance-covariance matrix of the five key factors in the model and the measure of literal intention-behaviour consistency, using an iterative maximum likelihood procedure provided by the program EzPath ${ }^{3}$. In the model tested, consistency is predicted from amount of reasoning and confidence, amount of reasoning is predicted from involvement and need for cognition, and confidence is predicted from consideration set size and involvement. In Figure 1 the model is presented, together with the path coefficients and standard deviations. A total of 11.8 per cent of variance in consistency is explained by its antecedents. All goodness of fit measures indicate that the model fits the data well, $\chi^{2}(6)=7.95, p=0.242, A G F I=0.92, \Gamma_{2}=0.98$, and $R M S R=0.06$. Path coefficients are not only significantly for the paths from amount of reasoning $(t=2.11, p<0.05)$ and confidence $(t=2.90, p<0.01)$ to consistency, but also for the paths between involvement and amount of reasoning $(t=$ $4.81, p<0.001)$, need for cognition and amount of reasoning ( $t=3.56, p<0.001)$, consideration set size and confidence $(t=6.06, p<0.001)$, and involvement and confidence $(t=3.92, p<0.001)$.

In addition, an alternative overall model was tested. It was tested whether the model in which the path between involvement and confidence is excluded, fits the data adequately. If this is the case, involvement only affects consistency through its impact on amount of reasoning, and not also through its effect on confidence. The simplified

\footnotetext{
2 The results presented here are further supported by regressing the decision moment on amount of reasoning and confidence. Decision moment was indicated by 136 respondents in a free-response format in wave 2 , and was coded as described in the method section. The multiple $R$ was $0.50, F(2,133)=21.98, p<0.001$. Both amount of reasoning and confidence were significantly and independently related to the decision moment (respective $t$ values were 2.29, and 5.64). Compared to others, people who had thought extensively about the elections, and those who were confident in their intention, as indicated in wave 1, knew significantly sooner which party to vote for, as indicated in wave 2 .

${ }^{3}$ EzPath is a program for causal modelling, and is comparable with LISREL and COSAN. Several indicators of overall fit of the model tested are provided, i.e. $\chi^{2}$, Adjusted Goodness of Fit (AGFI, Adjusted Population Gamma $\left(\Gamma_{2}\right)$, and Root Mean Square Residual (RMSR). As a global heuristic, good fit is indicated by a nonsignificant $\chi^{2}$, AGFI $>0.90, \Gamma_{2}>0.90$, and RMSR $<0.10$.
} 


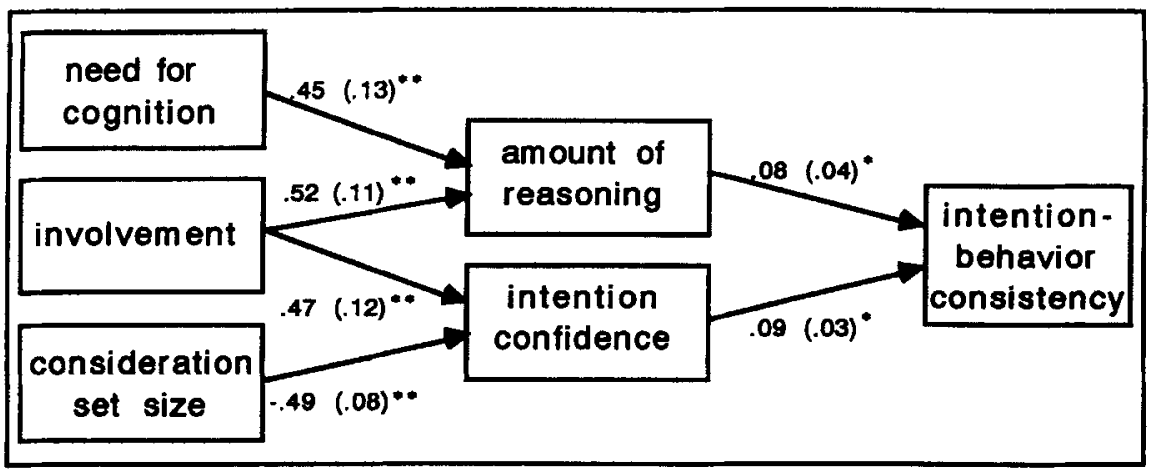

Figure 1. Antecedents of intention-behaviour consistency. Path coefficients with standard deviations between parentheses. ${ }^{*} p<0.05,{ }^{* *} p<0.001$

model was rejected, as is indicated by insufficient levels of the goodness of fit indices, $\chi^{2}(7)=22.26, p<0.002, A G F I=0.84, \Gamma_{2}=0.88$, and $R M S R=0.13$.

\section{DISCUSSION}

The results suggest that amount of reasoning and degree of confidence in one's intention independently moderate intention-behaviour consistency. Consistency is relatively high when individuals think and deliberate about the intended behaviour, and when they are confident in their intention. Consistency between voting intention and voting behaviour is very high in our study, as has been found in other studies on voting (e.g. Echebarria, Paez and Valencia, 1988; Granberg and Holmberg, 1990). Even with this high level of consistency the two moderators accounted for a significant amount of variance in consistency.

The moderating effect of involvement and need for cognition in the intentionbehaviour relationship, through their impact on amount of reasoning, indicates that explanations of attitude-behaviour consistency in terms of amount of cognitive effort expended during attitude formation (Chaiken, 1987; Petty and Cacioppo, 1986) do not exclusively hold for attitudes, but are also relevant for explaining intention-behaviour consistency. These explanations suggest that when an individual is motivated and able to engage in relatively effortful processing, underlying cognitive structures of an attitude become salient during the process of attitude formation, and are easily available at the time the person acts upon an attitude. Such may also be the case for intentions. Intentions that are readily available at the time the relevant decision has to be made are more likely to guide behaviour than intentions that are less available. Hence, factors that determine the availability of intentions, such as involvement and need for cognition, are likely to moderate intention-behaviour consistency. Bagozzi and Yi (1989), for example, manipulated subjects' opportunity to consider the perceived consequences of an act, and thus the degree to which they formed a behavioural intention. When intentions were well-formed, intention-behaviour consistency was stronger than when intentions were ill-formed. In the latter condition attitudes predicted behaviour directly.

Whereas the results concerning involvement, need for cognition, and intention- 
behaviour consistency extend what is known about the attitude-intention-behaviour relation, the results concerning consideration set size provide new insights that are particularly relevant for the intention-behaviour relation in a context of multiple alternatives. In a multiple choice context, the consideration set comprises the options that individuals actually will choose from. The present results show that consideration set size, through its impact on confidence in one's intention, affects intention-behaviour consistency over and above the effect of amount of reasoning. Large consideration sets, which are associated with less confidence in a particular intention, are related to less intention-behaviour consistency than smaller sets are. Processes related to consideration set size thus explain variance that cannot be explained by analysing intention-behaviour consistency along the traditional attitude-behaviour perspective. This demonstrates the usefulness of the concept of consideration set, when the behaviour of interest involves a multi-alternative choice situation.

It is important to stress the different roles that attitudes and intentions have in choice contexts such as the one examined in the present study. When people choose one alternative from a set of mutually exclusive alternatives, they can have positive attitudes toward more than one alternative, but they can have a choice intention with respect to only a single alternative. In other words, a positive attitude toward option $\mathrm{X}$ does not exclude a positive attitude toward option $\mathrm{Y}$, whereas an intention to choose $\mathrm{X}$ does exclude an intention to choose $\mathrm{Y}$. The size of the consideration set is a simple measure of the competition between alternatives that individuals experience. Larger sets are typically associated with more intensely experienced competition, leading to lower confidence in one's intention, and to less intention-behaviour consistency. Alternatively, a more detailed analysis of the impact of competing alternatives on intention-behaviour consistency is possible by measuring attitudes toward all alternatives that a person is aware of, rather than consideration set size. Such an analysis might reveal that both the number of alternatives considered, and their closeness in attitudinal strength to the alternative that a person intends to choose affect intentionbehaviour consistency. However, when people are confronted with a choice between a large set of alternatives, as in the case of Dutch elections or when choosing between brands of products, such an analysis requires extensive and relatively cumbersome procedures of measurement and statistical analysis. Moreover, our results show that simply knowing the number of alternatives that people consider for choice already provides significant information about the likelihood that their intentions predict their behaviour. Nevertheless, future research may examine the role of competing alternatives in intention-behaviour consistency in more detail.

The study presented here is limited in several aspects. First, the data are correlational. Hence, any causal interpretation of the present results should be done with care. This especially holds for mutual relations among variables that were measured simultaneously in the questionnaire (wave 1). For instance, the design prevents exclusion of the possibility that consideration set size reflects differences in intention and confidence, as was suggested by a reviewer: a low intention and low level of confidence may instigate an individual to search for alternatives, thus resulting in a large consideration set. However, the present model, including the indicated relationships, is the best fitting model to these data on empirical grounds. Alternative formulations of the mutual relations, as far as these are equally parsimonious, result in significantly less well fitting models. Nevertheless, experimental designs in future research are necessary to examine causal relationships in detail. Second, the mail questionnaire poses restric- 
tions on the type and amount of questions that can be included. As a consequence, some of the concepts were measured less adequately. A single item measure of confidence was used, while the involvement and amount of reasoning measures were composed of two items each. Although the predicted relations were found, multiple measures of these constructs are recommendable in future research. Still, the most likely result of having only a few indicators for a construct is that the lower reliability attenuates true effects. Despite such a possible attenuation, our hypotheses are confirmed, and consistent effects of the proposed moderating variables are found.

The use of self-reports of voting behaviour may lead to bias, if respondents overreport to have voted in correspondence with their intention, in order to present themselves in a favourable way. Such biasing effects of self-presentation can be expected in particular if one is committed to a particular party, for instance because of party membership or a history of consistent voting for this party (Crosby and Taylor, 1983). We do not think that self-presentation motives have been salient in our study, as it is unlikely that respondents in wave 2 have suspected a link between the two measures: wave 1 comprised a mail survey, presented as an election study by a university, whereas wave 2 comprised a 10-minute telephone interview by a marketing company, in which voting behaviour was only one item ${ }^{4}$. Also, a 'bandwagon' effect, in which voters report to have voted for a winning party, while in fact they did not vote, or voted for another party (e.g. Sudman and Bradburn, 1982), is unlikely to be present in our study, because none of the parties won considerably in these elections.

The present study shows two distinct effects of involvement. First, involvement affects the amount of reasoning, and thus has an effect on intention-behaviour consistency. Second, involvement affects the degree of confidence in one's intention, which in turn moderates consistency. The first effect encompasses processes that take place during intention formation, and is well documented in dual-process models of attitude formation and change (Chaiken, 1987; Petty and Cacioppo, 1986). The second effect encompasses processes during intention implementation (cf. Gollwitzer, 1993). This effect, referring to the consequences of having taken a position, has received less research attention so far. Our results suggest that both aspects of involvement are distinct moderating factors in the intention-behaviour relationship, and therefore deserve further attention. Finally, the results of the present study illustrate the relevance of considering multiple moderators in research on the relationships between attitudes, intentions, and behaviours.

\section{REFERENCES}

Ajzen, I. and Fishbein, M. (1977). 'Attitude-behavior relations: A theoretical analysis and review of empirical research', Psychological Bulletin, 84: 888-918.

Ajzen, I. and Fishbein, M. (1980). Understanding Attitudes and Predicting Social Behavior, Prentice-Hall, Englewood Cliffs, NJ.

Bagozzi, R. P. and Warshaw, P. R. (1990). 'Trying to consume', Journal of Consumer Research, 17: $127-140$.

Bagozzi, R. P. and Yi, Y. (1989). 'The degree of intention formation as a moderator of the attitude-behavior relation', Social Psychology Quarterly, 52: 266-279.

${ }^{4}$ Even if intention-behaviour consistency is inflated due to this bias, the variation restriction in our dependent measure would make it only more difficult to observe the moderating effects that we did find. 
Belk, R. W. (1985). 'Issues in the intention-behavior discrepancy'. In: Sheth, J. N. (Ed.) Research in Consumer Behavior, Vol. 1, JAI Press Inc., Greenwich, CO.

Bentler, P. M. and Speckart, G. (1979). 'Models of attitude-behavior relations', Psychological Review, 86: 452 464.

Bettman, J. R. (1979). An Information Processing Theory of Consumer Choice, Addison-Wesley, Reading, MA.

Bettman, J. R. and Park, C. W. (1980). 'Effects of prior knowledge and experience and phase of the choice process on consumer decision processes: A protocol analysis', Journal of Consumer Research, 7: 234-248.

Cacioppo, J. T. and Petty, R. E. (1982). 'The need for cognition', Journal of Personality and Social Psychology, 42: 116-131.

Cacioppo, J. T., Petty, R. E. and Kao, C. F. (1984). 'The efficient assessment of need for cognition', Journal of Personality Assessment, 48: 306-307.

Cacioppo, J. T., Petty, R. E., Kao, C. F. and Rodriguez, R. (1986). 'Central and peripheral routes to persuasion: An individual difference approach', Journal of Personality and Social Psychology, 51: 1032-1043.

Chaiken, S. (1987). 'The heuristic model of persuasion'. In: Zanna, M. P., Olson, J. M. and Herman, C.P. (Eds) Social Influence: The Ontario Symposium, Vol. 5, Erlbaum, Hillsdale, NJ.

Crosby, L. A. and Taylor, J. R. (1983). 'Psychological comment and its effects on post-decision evaluation and preference stability among voters', Journal of Consumer Research, 9: 413-431.

Davidson, A. R. and Jaccard, J. J. (1979). 'Variables that moderate the attitude-behavior relation: Results of a longitudinal survey', Journal of Personality and Social Psychology, 37: 1364-1376.

Echebbaria, A., Paez, D. and Valencia, J. F. (1988). 'Testing Ajzen and Fishbein's attitudes model: The prediction of voting', European Journal of Social Psychology, 18: 181-189.

Fazio, R. H. (1990). 'Multiple processes by which attitudes guide behavior: The mode model as an integrative framework', Advances in Experimental Social Psychology, 23: 75-109.

Fazio, R. H. and Zanna, M. P. (1978). 'On the predictive validity of attitudes: The role of direct experience and confidence', Journal of Personality, 46: 228-243.

Gertzen, H. (1992). 'Component processes of phased decision strategies', Acta Psychologica, 80: 229-246.

Gollwitzer, P. M. (1993). 'Goal achievement: The role of intentions', European Review of Social Psychology, 4: 141-185.

Granberg, D. and Holmberg, S. (1990). 'The intention-behaviour relationship among U.S. and Swedish voters', Social Psychology Quarterly, 53: 44-54.

Howard, J. A. and Sheth, J. N. (1969). The Theory of Buyer Behavior, Wiley, New York.

Krosnick, J. A. (1988). 'The role of attitude importance in social evaluation: A study of policy preferences, presidential candidate evaluations, and voting behavior', Journal of Personality and Social Psychology, 55: 196-210.

Petty, R. E. and Cacioppo, J. T. (1986). Communication and Persuasion: Central and Peripheral Routes to Attitude Change, Springer-Verlag, New York.

Petty, R. E., Cacioppo, J. T. and Schumann,D. (1983). 'Central and peripheral routes to advertising effectiveness: The moderating role of involvement', Journal of Consumer Research, 10: 135-146.

Sheppard, B. H., Hartwick, J. and Warshaw, P. R. (1988). 'The theory of reasoned action: A meta-analysis of past research with recommendations for modifications and future research', Journal of Consumer Research, 15: 325-343.

Sherif, M. and Hovland, C. I. (1961). Social Judgment: Assimilation and Contrast Effects in Communication and Attitude Change, Yale University Press, New Haven, CO.

Sudman, S. and Bradburn, N. M. (1982). Asking Questions, Jossey-Bass Publishers, San Francisco.

Verplanken, B. (1989). 'Involvement and need for cognition as moderators of beliefs-attitudeintention consistency', British Journal of Social Psychology, 28: 115-122.

Warshaw, P. R. and Davis, F. D. (1985). 'Disentangling behavioral intention and behavioral expectation', Journal of Experimental Social Psychology, 21: 213-228. 